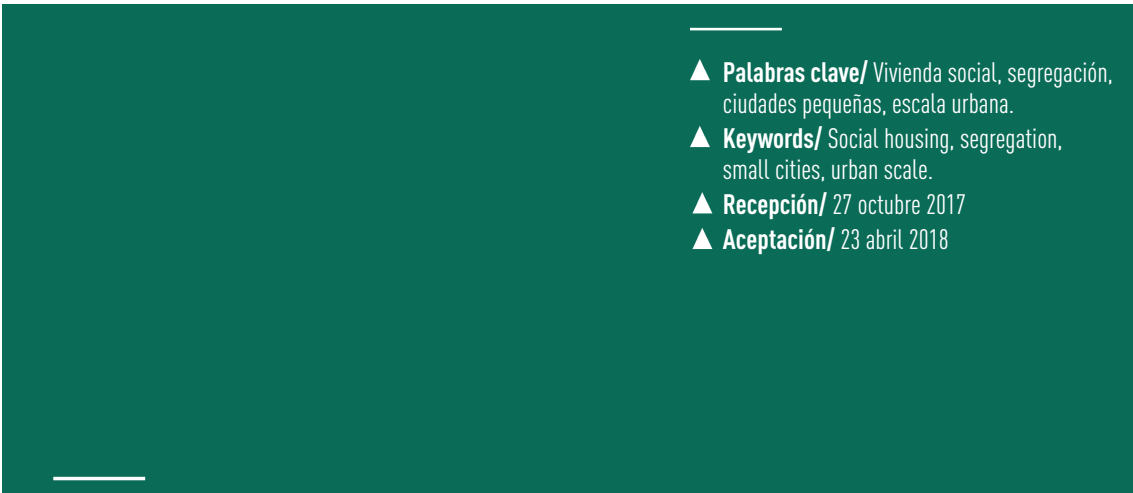

\title{
Vivienda social y segregación: Una aproximación para entender las diferencias entre grandes y pequeñas ciudades ${ }^{1}$
}

\author{
Social Housing and Segregation: \\ An approach to understanding the difference \\ between large and small cities ${ }^{1}$
}

Luis Vergara

Profesor de Historia y Geografía, Universidad de la Frontera, Chile.

Magister en Ciencias Sociales Aplicadas,

Universidad de la Frontera, Chile.

Investigador Invitado, Real Estate \& Planning, University of Reading, Inglaterra.

llvergara@uc.cl

RESUMEN/ Este trabajo critica la perspectiva especialista que plantea que la segregación urbana es per se menos maligna en las ciudades pequeñas. A través de entrevistas semi-estructuradas y observación no participante aplicadas en dos barrios de vivienda social segregados que se localizan en ciudades de distinto tamaño, se argumenta a favor de una aproximación que reconozca que la malignidad de la segregación no solo se explica por factores de orden espacial, sino que también por aspectos vinculados a la política de movilidad y la dotación de servicios y equipamientos público-privados que posee la periferia urbana. Se concluye que esta aproximación contribuiría a entender de mejor manera los efectos de la política de vivienda social en ciudades de diferente tamaño. ABSTRACT/ This work is a critique to specialized approaches according to which urban segregation is per se less pernicious in small cities. Through semi-structured interviews and non-participant observation applied in two quarters of segregated social housing in cities of different sizes, the argument is made in favor of an approach that acknowledges that the perniciousness of segregation is not only explained by spatial factors but by aspects linked to the policy of mobility and public-private services and infrastructure in urban peripheries. The conclusion is reached that this approach will help better understand the impacts of social housing policies in cities of different sizes.

INTRODUCCIÓN. El modelo de vivienda social chileno ha sido tremendamente exitoso desde el punto de vista cuantitativo (Ducci 1997). El déficit habitacional se ha reducido desde 841.772 a 391.545 viviendas entre 1983 y 2015 , lo que representa una disminución del $53,5 \%$ en un período en el cual la población nacional aumentó en cerca de 6,3 millones. Sin embargo, la masividad de la construcción ha estado acompañada de un grave problema: el de la segregación. Es decir, la concentración espacial, el aislamiento social y la estigmatización de las familias de más bajos ingresos (Sabatini 2006). La literatura local se ha 옹 referido a esto como el "problema de los con techo" (Rodríguez y Sugranyes 2004), concepto que enfatiza la malignidad de la segregación, ya que aún cuando las familias poseen una vivienda, tienen serios problemas para acceder a los recursos funcionales y simbólicos de les brinda habitar en la ciudad.

El problema de la segregación ciertamente no se vincula solo a la masividad de la política habitacional, sino que también a la operatoria no regulada del mercado de suelo, el cual se ajusta permanentemente al alza según la capacidad de los mejores pagadores por zona (Sabatini, Sarella y Vásquez 2009). El resultado ha sido una ciudad que concentra espacialmente a la vivienda social en la periferia, allí donde e valor por metro cuadrado es más bajo. Pero un aspecto es la segregación espacial y otro diferente son las consecuencias sociales de dicho fenómeno. Diferentes trabajos han concluido que la segregación espacial de la vivienda social es especialmente maligna en las grandes ciudades. La estigmatización, la inacción tanto en jóvenes como en adultos y el abandono escolar son síntomas de la guetificación de muchos conjuntos de vivienda social segregados en áreas metropolitanas (Sabatini, Wormald, Sierralta y Peters 2010). En cambio, aunque igualmente en condición segregada, los conjuntos de vivienda social de ciudades 
pequeñas no parecen estar experimentando el mismo fenómeno. En estas ciudades la vivienda social parece actuar como un mecanismo que contribuye a mejorar la calidad de vida de las personas (Vergara y Garín 2016; Hidalgo y Marquardt 2011). ¿Qué explica la diferencia entre la 'malignidad' de la segregación de la vivienda social en las ciudades grandes y pequeñas? La respuesta que ha dado la literatura a ello es el tamaño de la ciudad (Sabatini et al. 2010; Vergara y Garín 2016). Parece ser que cuanto más pequeña es la ciudad, la segregación en ella adquiere un carácter más 'amigable' y, por tanto, sería incluso deseable redirigir las políticas de vivienda social hacia este tipo de lugares, siempre y cuando garanticen fuentes laborales para sus habitantes (Zunino et al. 2011).

Este trabajo busca analizar los mecanismos que explican la diferencia da la malignidad en la segregación de la vivienda social entre ciudades grandes y pequeñas.

Se argumenta a favor de la necesidad de ampliar la discusión respecto a los factores que median la malignidad de la segregación, planteando que las diferencias entre ciudades no se explican solo por factores de orden espacial, sino que también por decisiones políticas y económicas. Específicamente, la malignidad de la segregación estaría vinculada a factores espaciales, pero también a las políticas de movilidad y la dotación de servicios y equipamientos público-privados de la periferia urbana. Dicho argumento se despliega a través de la mirada comparada de dos barrios de vivienda social localizados en zonas periféricas y con segregación a gran escala, pero en ciudades de diferente tamaño: Juvencio Valle en Santiago y Villa Las Naciones en Angol.

\section{LA MALIGNIDAD DE LA SEGREGACIÓN: ¿UNA CUESTIÓN DE} ESCALA?

la discusión sobre la segregación se produjo en la década de 1980, con el giro espacial de las ciencias sociales. Hasta ese entonces, la segregación era entendida mayoritariamente como un fenómeno estructuralmente determinado y vinculado profundamente a las desigualdades macroeconómicas de los países. Desde esta aproximación, denominada estructuralista, el espacio prácticamente no tenía cabida como factor explicativo de la segregación. Frente a ello, Edward Soja y el concepto de dialéctica socio-espacial (1980), intentan reposicionar el espacio como un aspecto determinante en la explicación de los fenómenos sociales. Pero la discusión va más allá e incorpora la noción de escala, buscando entender cómo es que los fenómenos sociales operan de manera diferenciada a través de las diferentes escalas territoriales (Massey 1993).

El giro espacial impulsó, entre otras cosas, estudios para medir la segregación en ciudades de diferentes tamaños o escalas. Un trabajo pionero en esta línea fue el de Farley (1991), quien creó un ranking de segregación racial en ciudades norteamericanas. Dicho trabajó concluyó que las ciudades grandes presentaban niveles de segregación racial más altos que las ciudades pequeñas. Trabajos cuantitativos similares han sido desarrollados en latinoamérica. Por ejemplo, Sabatini et al. (2010), aplicando índices de homogeneidad y aislamiento espacial en diferentes ciudades chilenas, plantean que la segregación a gran escala -característica de las ciudades grandes- es más maligna que la de ciudades pequeñas que se caracterizan por tener segregación espacial a escala más reducida. Desde una perspectiva más cualitativa y centrados en estudios de casos, algunos trabajos reafirman la idea de que en las ciudades pequeña la segregación es menor. Zunino et al. (2011), analizando un barrio de viviendas sociales en Pucón, mostraron que, aunque este estaba segregado en la periferia de la ciudad, la satisfacción residencial de sus habitantes era alta. Una de las razones que explica los resultados, arguyen los autores, es el tamaño de la ciudad y el estilo de vida de 'ciudad chica'

Pero la idea de que la segregación es menos maligna en las ciudades pequeñas ha sido recientemente cuestionada. Parece ser también que en algunos autores dicha relación se sostiene en una visión romántica de las ciudades pequeñas. La literatura sobre ciudades no-metropolitanas ha caracterizado estos lugares en oposición a las grandes ciudades. Por ejemplo, Bellet y Llop (2004) se refieren a estas ciudades como más sustentables, más cohesionadas, menos segregadas y mejor conectadas que las áreas metropolitanas. Las ciudades pequeñas y metropolitanas experimentan los mismos fenómenos sociales de formas muy diferentes, debido a que su tamaño redefine su naturaleza. Una aproximación de este tipo implica cierto fundamentalismo espacial, el cual podría contribuir a 'naturalizar' los problemas sociales que ocurren en las pequeñas ciudades. También en una crítica a la relación escala-segregación, Krupka (2007) ha señalado que esta es espuria. El problema, argumenta el autor, es estadístico y recae en el tamaño de las áreas en las que se mide la segregación. Es lo que se conoce como "el problema de la grilla", el cual sería 
inherente a cualquier indicador que intente evaluar espacialmente la segregación urbana a diferentes escalas. En efecto, por extensión urbana es mucho más probable que los distritos urbanos que componen las ciudades se muestren más heterogéneos en las ciudades pequeñas que en las grandes (ver también Sabatini et al. 2010). Ello, como Krupka (2007) argumenta, no es suficiente para concluir que las ciudades pequeñas son menos segregadas per se que las grandes solo por una cuestión de tamaño. La relación parece estar medida por otro tipo de mecanismos, difíciles de discernir a través de la aplicación de índices. Finalmente, Ruiz-Tagle y López (2014) han argumentado que la relación entre la escala y la segregación no es mecánica, sino que más bien depende de lo que ellos llaman la "equivalencia espacial neoliberal". Esto quiere decir que, la malignidad de la segregación se vincula a la manera en que instituciones poderosas -como el Estadointervienen sobre los lugares segregados, favoreciéndolos o restándoles el acceso a oportunidades urbanas a quienes habitan en ellos.

\section{ESTRATEGIA METODOLÓGICA Y CASOS DE ESTUDIO: Una mirada a dos barrios en entornos segregados.}

Para la estrategia metodológica se

consideraron tres elementos de base.

Primero, que el origen de la relación espuria entre la malignidad de la segregación y el tamaño de la ciudad se encuentra en el uso de indicadores espaciales (Krupka 2007). Segundo, que hay una necesidad de entender la segregación no solo como un problema espacial, sino que especialmente social (Saraví 2008). Y tercero, que existe escasez de trabajos cualitativos que analicen comparativamente la situación de segregación entre ciudades de diferente tamaño. En función de esto, este trabajo se sustenta en una metodología cualitativa, basada en la observación no-participante y entrevistas semiestructuradas a 25 personas en dos barrios de vivienda social localizados en sectores periféricos de ciudades de tamaños radicalmente distintos: Angol y Santiago. En cada uno de los barrios el acceso a la muestra se realizó a partir de la técnica de muestreo conocida como bola de nieve.
Angol corresponde a una ciudad pequeña, de cerca de 50.000 habitantes que se localiza en La Región de la Araucanía, en el sur de Chile. Esta es una ciudad que destaca permanentemente a nivel nacional por presentar altos índices de pobreza y cesantía, pero que en las últimas dos décadas casi ha duplicado su superficie urbana, situación que ha estado sustentada especialmente en la construcción de viviendas sociales. En esta ciudad se emplea como caso de estudio a Villa las Naciones, un conjunto de vivienda social construido entre 2003 y 2007, localizado en la periferia de la ciudad y rodeado de otros conjuntos de vivienda social.

En el caso de Santiago, el caso de estudio fue Juvencio Valle. Este vecindario fue construido en 2009 y se ubica en la periferia sur de Santiago, específicamente en la comuna de San Bernardo. Aunque Juvencio Valle fue pensado como un barrio de integración social, en este lugar habitan mayoritariamente familias de clase media emergente. El barrio se inserta en un entorno rodeado por conjuntos de vivienda social, con altas tasas de delitos y poco dinamismo inmobiliario (figura 1).
CIUDAD: SANTIAGO

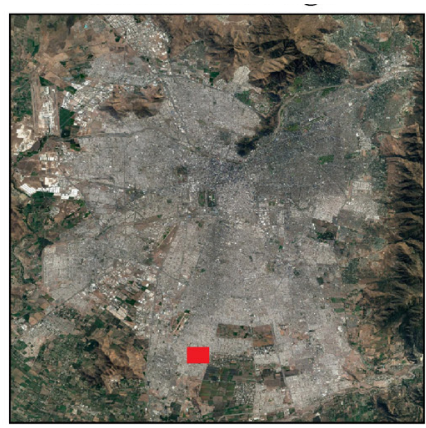

CIUDAD: ANGOL

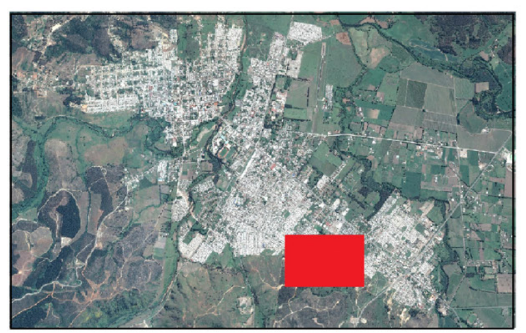

BARRIO Y ENTORNO

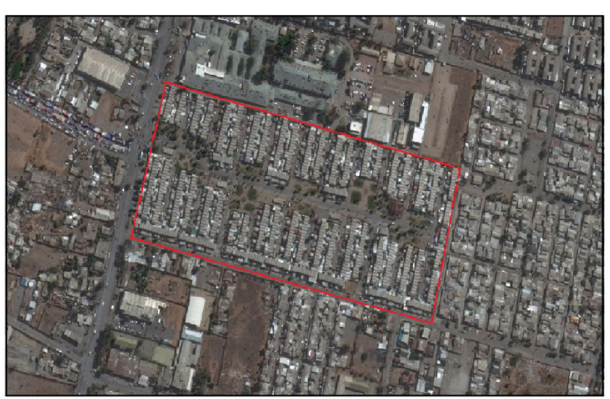

BARRIO Y ENTORNO

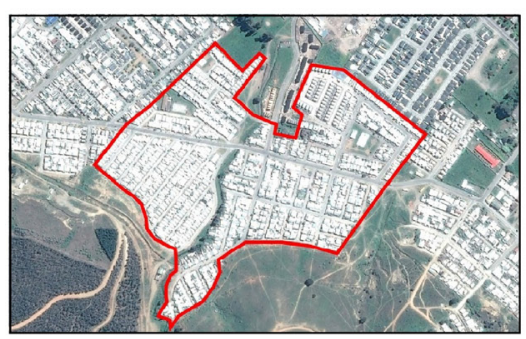

VILLA JUVENCIO VALLE

Figura 1. Casos de estudio: Juvencio Valle en Santiago y Villa Las Naciones en Angol (fuente: Elaboración propia). 


\section{LA MAGNITUD ESPACIAL DE LA SEGREGACIÓN Y LA DIVERSIDAD DEL ENTORNO AL BARRIO.}

aspecto que media la relación entre escala urbana y malignidad de la segregación, es la magnitud espacial de la segregación. Esto es, qué tan espacialmente extensa es la homogeneidad social del área en la cual se inserta el barrio.

Los habitantes de Juvencio Valle entienden que la segregación a gran escala es más común en las grandes ciudades, lo que implica más problemas sociales. Justamente reconocen que el barrio se inserta en un entorno socioeconómicamente homogéneo, donde hay mayoritariamente viviendas de origen estatal o campamentos. Los habitantes establecen una relación intrínseca entre la homogeneidad socioeconómica del área y la inseguridad, que los lleva a percibir la zona donde se inserta el vecindario como muy insegura. Eso se refleja en la actitud de desconfianza permanente hacia lo que se encuentra fuera del barrio, situación que se ha materializado en el enrejamiento del vecindario y de los pasajes que en él existen (imagen 1).

El caso de Angol es distinto, por cuanto los habitantes de Villa las Naciones (imagen 2) no perciben problemas sociales vinculados a la segregación. De hecho, plantean que la segregación en la ciudad se manifiesta con una naturaleza muy diferente a la de las grandes ciudades como Santiago, ya que aunque las viviendas sociales se localicen en zonas periféricas, siempre es posible encontrar familias de mejor condición socioeconómica a poca distancia. Si bien dicha condición de heterogeneidad no garantiza relaciones sociales de carácter pluriclasistas, sí parece estar asociada a la inexistencia de problemas sociales vinculados a la segregación. La segregación y los problemas que esta puede estar generando, no parecen tener vinculación para los habitantes de ciudades que tienen mayor nivel de heterogeneidad social en el entorno.

Estos resultados muestran una diferencia fundamental entre las ciudades grandes y pequeñas. En las primeras, la escala espacial de la segregación parece ser mayor, y ello se vincula a mayores problemas sociales y especialmente a una mayor inseguridad. Estos resultados apoyan la idea de que el tamaño de la ciudad tiene un efecto sobre la malignidad percibida de la segregación y, por tanto, el espacio tiene un rol sobre este

\section{fenómeno social.}

¿AFECTA EL TAMAÑO DE LA CIUDAD EN EL ACCESO A OPORTUNIDADES

\section{URBANAS?} tiene una superficie urbana de 866 ha,

Santiago se empina por las 83.000 ha. Más allá de esta diferencia en el tamaño, ¿cómo funciona la escala para quienes habitan en conjuntos de vivienda social en ambas ciudades? La respuesta a esta interrogante, que tiene que ver con la distancia que tienen sus barrios con el centro de la ciudad, da cuenta de diferencias abismantes. Mientras la distancia que une a los distintos barrios de vivienda social con el centro de Angol varía entre los 800 metros a los 4 kilómetros, la distancia entre Juvencio Valle y el centro de Santiago es de 20 kilómetros. Es decir, la vivienda social en Angol se localiza en una mejor posición con respecto al centro de la ciudad que en el caso de Santiago. Así es percibido también por los habitantes de los barrios. Mientras en Villa Las Naciones la distancia al centro no es vista como un problema, sí lo es para los residentes de Juvencio Valle, quienes se sienten lejanos del centro de la ciudad. Hasta aquí el tamaño de la ciudad y la distancia respecto del centro parecieran configurar una relación directa con la segregación. Sin embargo, consultados respecto a los inconvenientes que les genera vivir lejos del centro, una parte de los habitantes de Juvencio Valle sostiene que la distancia del centro no necesariamente actúa como un obstáculo en el acceso a oportunidades urbanas, ya que el entorno está bien equipado y servido. Esto último tiende a matizar el rol que la escala de la ciudad juega sobre la malignidad de la segregación en ciudades grandes.

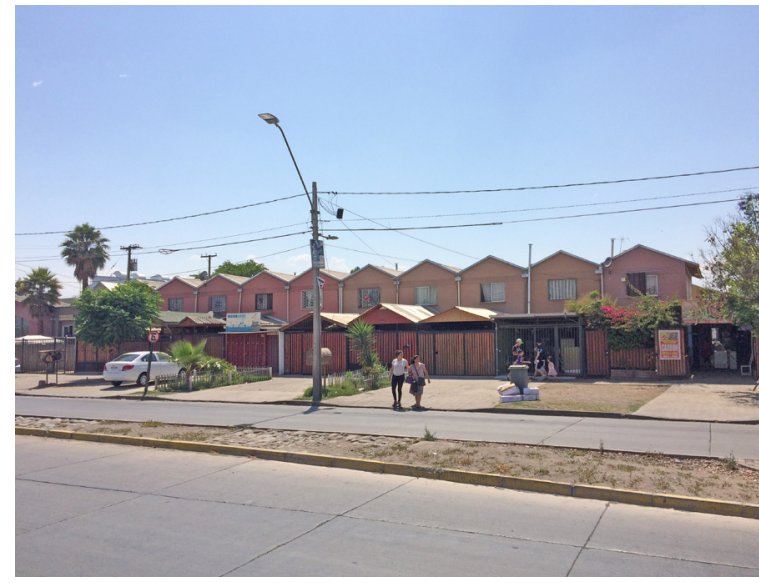

Imagen 1. Calles internas enrejadas, Juvencio Valle (fuente: $E$ ! autor 2015.

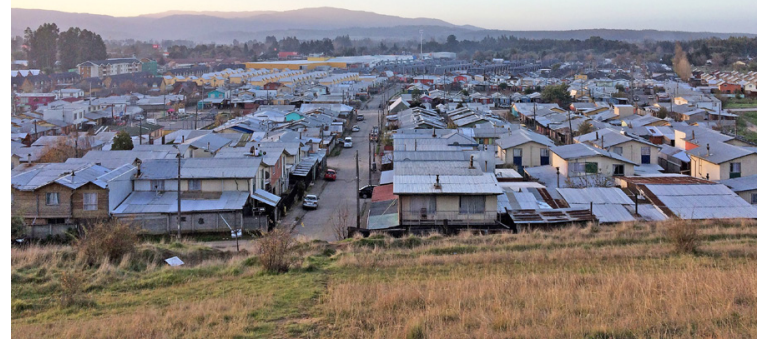

Imagen 2. Panorámica Villa las Naciones y barrios circundantes (fuente: El autor 2017). 
CONCLUSIÓN. En este artículo se analizó comparativamente los elementos que explican la malignidad de la segregación a partir de la perspectiva de los habitantes de vivienda social en dos ciudades de diferente tamaño. Los resultados cuestionan las perspectivas espacialistas que sostienen que cuanto más pequeña es la ciudad, menos maligna es la segregación. Si bien se reconoce que el tamaño de la ciudad influye en la malignidad a través de la extensión espacial de la homogeneidad y la distancia desde el barrio al centro de la ciudad, hay factores de orden político (políticas de movilidad) y de inversión público-privada (grado de gentrificación periférica) que también tienen influencia sobre este fenómeno social y contribuyen a matizar la idea de que las ciudades pequeñas son menos segregadas. La movilidad y la gentrificación son así mecanismos específicos a través de los cuales opera la equivalencia espacial neoliberal (Ruiz-Tagle y López 2014) en las ciudades chilenas. Reducir la discusión al tamaño de la ciudad podría conllevar un peligro en la toma de decisiones sobre políticas de vivienda. Las ciudades pequeñas no necesariamente son mejores que las grandes áreas metropolitanas, y una reorientación de la vivienda hacia ellas podría tener consecuencias nefastas para sus habitantes y la dinámica urbana que allí se desarrolla. Por lo tanto, las aproximaciones netamente espaciales de la segregación deben ser complementadas con una aproximación que centre su análisis en las políticas de regulación e inversión público-privada que tienen las ciudades. Esto podría mejorar de forma importante los resultados sociales de la vivienda construida por políticas estatales, no solo en ciudades pequeñas, sino que también en las grandes áreas metropolitanas.

\section{REFERENCIAS}

Bellet C y Llop J 2004 "Miradas a otros espacios urbanos: las ciudades intermedias" Scripta Nova, 8, 165 Ducci, M., 1997. "Chile: el lado obscuro de una politica de vivienda exitosa." EURE, 23 (69): 99-115. Farley, R., 1991. "Residential segregation of social and economics groups among blacks, 1970-1980." En Jencks, C. y Peterson, P. (Eds.). The Urban underclass. Washington: Brookings Institution, 274-298. Krupka, D., 2007. "Are Big Cities More Segregated? Neighbourhood Scale and the Measurement of Segregation." Urban Studies, 44 (1): 187-197.

Massey, D., 1993. "Power-Geometry and progressive sense of place." En Bird, J., Curtis, B., Putnam, T., Robertson, G. y Tickner, L, Mapping the Futures: Local Cultures, Global Change. Routledge: London, 60-70 Rodriguez, A. y Sugranyes, A., 2004. "El problema de vivienda de los "con techo"." EURE, 30 (91): 53-65. Ruiz-Tagle, J. y López, E., 2014. "Estudio de la segregación residencial en Santiago de Chile: revisión crítica de algunos problemas metodológicos y conceptuales." EURE, 40, (19): 25-48

Sabatini, F., 2006. La segregación social del espacio en las ciudades de América Latina. Banco Interamericano de Desarrollo.

Sabatini, F., Sarella, M, y Vásquez, H., 2009. "Gentrificación sin expulsión, o la ciudad latinoamericana en una encrucijada histórica." Revista 180, 24, 18-25.
Sabatini, F., Wormald, G., Sierralta., C. y Peters, P., 2010. "Segregación Residencial en Santiago: Tendencias 1992-2002 y efectos vinculados con su escala Geográfica". En Sabatini, F., Salcedo, R., Wormald, G. y Cáceres, G., Tendencias de la segregación en las principales ciudades Chilenas: Análisis Censal 1982-2002. Santiago de Chile: Universidad Católica e Instituto Nacional de Estadísticas, 19-42. Saravi, G., 2008. "Mundos aislados: Segregación urbana y desigualdad en la ciudad de México." EURE, 34 (103): 93-110.

Soja, E., 1980. "The socio-spatial dialectic." Annals of the Association of American geographers, 70 (2): 207-225

Vergara, L. y Garin, A., 2016. "Vivienda social y segregación socioespacial en una ciudad pequeña: El caso de Angol, Chile." Polis, 15, (44): 457-486.

Zunino, H., Hidalgo, R., y Marquardt, E., 2011. "Vivienda Social y segregación espacial en la ciudad de Pucón. Chile: Entre el enclaustramiento y la integración con el hábitat turistico." INVI, 26 (71): 15-55. 